\title{
Effect of UV Irradiation on the Antioxidant and Anti-adipogenic Activities of Quercetin
}

\author{
Da-Jeong Heo, Myung-Soo Shon, Gyo-Nam Kim, and Seung-Cheol Lee
}

Received March 18, 2014; revised July 22, 2014; accepted July 24, 2014; published online February 28, 2015

(C) KoSFoST and Springer 2015

\begin{abstract}
Quercetin is one of the most abundant flavonol in the human diet and it is found in various plants. Although many of the biological activities of quercetin have been well described, the effect of UV irradiation on its physiological function is still relatively unknown. In this study, we compared the antioxidant and anti-adipogenic activities of intact and UV-irradiated quercetins. The UV irradiation significantly and markedly attenuated the antioxidant activity of quercetins, as determined by the DPPH radical scavenging assay. However, the UV irradiation had little effect on its anti-adipogenic activity, which was determined using 3T3-L1 adipocyte differentiation model. Taken together, these results suggest that the antioxidant and anti-adipogenic activities of quercetin are independently modulated through molecular and chemical based mechanisms.
\end{abstract}

Keywords: quercetin, UV irradiation, antioxidant, antiadipogenic

\section{Introduction}

Quercetin (3,3',4',5,7-pentahydroxyflavone), a plant pigment flavonoid, is widely distributed in many fruits, flowers, and vegetables. Previous studies have shown that quercetin exhibits antioxidant, anti-carcinogenic, anti-inflammatory, and antiviral activities and plays a role in the prevention of platelet aggregation (1-4). Quercetin has also been shown to exert an anti-obesity effect via the regulation of adipogenesis, which in occurs through the up-regulation of

Da-Jeong Heo, Myung-Soo Shon, Gyo-Nam Kim, Seung-Cheol Lee $(\bowtie)$ Department of Food Science and Biotechnology, Kyungnam University, Changwon, Gyeongnam 631-701, Korea

Tel: +82-55-249-2684; Fax: +82-55-249-2995

E-mail: sclee@kyungnam.ac.kr the adenosine monophosphate-activated protein kinase (AMPK) signaling pathway in 3T3-L1 preadipocytes. In addition, it causes apoptosis of mature adipocytes via the suppression of extracellular signal-regulated kinases (ERKs) and c-Jun N-terminal kinase (JNK) phosphorylation (5).

Quercetin is known to prevent UV induced oxidation. However, UV irradiation induces significant structural changes in quercetin, which have been shown to subsequently decrease its antioxidant activity (6). In this study, we report preliminary results for the effects of UV irradiation on the antioxidant and anti-adipogenic activities of quercetins.

\section{Materials and Methods}

Reagents Quercetin hydrate was purchased from Acros Organics (Morris Plains, NJ, USA). Oil Red O, insulin, dexamethasone, isobutylmethylxanthine (IBMX), and DPPH were purchased from Sigma-Aldrich (St. Louis, MO, USA). Dulbecco's modified Eagle's medium (DMEM), fetal calf serum (FCS), fetal bovine serum (FBS), penicillin, and streptomycin were purchased from Gibco BRL (Carlsbad, CA, USA). Unless otherwise noted, all other chemicals were purchased from Sigma-Aldrich.

UV irradiation A $10 \mathrm{mM}$ stock solution of quercetin was freshly prepared in methanol immediately before the experiments. For all the experiments, a dilution of the stock quercetin solution was freshly prepared using methanol and used. Quercetin (100 $\mu \mathrm{M}$ in methanol) was irradiated in UV irradiation apparatus (UV-2X; Dongseo Science Co., Ltd., Seongnam, Gyeonggi, Korea) equipped with two UV lamps (15 W, G15T8-AN; Sankyo Denki, Kanagawa, Japan) that emit UV rays at $253.7 \mathrm{~nm}$. The samples, placed in quartz cuvettes $(1 \times 1 \times 4.5 \mathrm{~cm})$, were placed at a distance of $10 \mathrm{~cm}$ from the lamps and irradiated for $3 \mathrm{~h}$. 
Absorption spectra of quercetin The absorption spectra of quercetin were determined using a UV-visible spectrophotometer (Optizen pop; Mecasys Co., Ltd., Daejeon, Korea). The absorbance of all samples was recorded at every $1 \mathrm{~nm}$ between 200 and $500 \mathrm{~nm}$, both before and after UV irradiation.

DPPH radical scavenging activity (RSA) The DPPH RSA of quercetin was determined according to the method of Jeong et al. (7). The sample $(0.1 \mathrm{~mL})$ was mixed with $0.9 \mathrm{~mL}$ of $0.041 \mathrm{mM}$ DPPH in ethanol and incubated for $30 \mathrm{~min}$. The absorbance of the sample was then measured at $517 \mathrm{~nm}$ using a spectrophotometer (UV-1601; Shimadzu, Kyoto, Japan). RSA was expressed in terms of percentage inhibition and calculated using the following formula:

$$
\% \text { DPPH RSA }=\left[1-\left(\mathrm{A}_{\text {sample }} / \mathrm{A}_{\text {control }}\right)\right] \times 100
$$

where $\mathrm{A}_{\text {control }}$ is the absorbance of the control reaction $(0.1$ $\mathrm{mL}$ of distilled water and $0.9 \mathrm{~mL}$ of DPPH), and $\mathrm{A}_{\text {sample }}$ is the absorbance of the sample.

3T3-L1 cell culture and anti-adipogenic activity 3T3L1 cells were purchased from American Type Culture Collection (ATCC, Rockville, MD, USA). The cells were cultured in DMEM supplemented with $10 \%(\mathrm{v} / \mathrm{v}) \mathrm{FCS}$, $100 \mathrm{U} / \mathrm{mL}$ penicillin, and $100 \mu \mathrm{g} / \mathrm{mL}$ streptomycin. Adipogenesis and the Oil Red O staining for qualitative and quantitative analyses of intracellular lipids were carried out as described previously (8). Briefly, preadipocytes were grown until confluence. At $2 \mathrm{~d}$ post-confluence (designated as day 0 ), the $3 \mathrm{~T} 3-\mathrm{L} 1$ cells were stimulated to differentiate with $10 \%$ FBS-DMEM supplemented with the standard adipogenic cocktail ( $167 \mathrm{nM}$ insulin, $0.25 \mathrm{mM}$ dexamethasone, and $0.5 \mathrm{mM}$ IBMX). After $2 \mathrm{~d}$ of differentiation, the medium was changed to $10 \%$ FBS-DMEM with $167 \mathrm{nM}$ insulin only. On day 4 , the medium was changed to $10 \%$ FBS-DMEM with no additional supplements and the cells were incubated until day 7. The 3T3-L1 cells were treated with quercetin or UV-irradiated quercetin for 4 days (day 2-day 2).

Statistical analysis All data are presented as mean \pm SD. Statistical analysis was performed using the Statistical Package for Social Science (SPSS version 14.0; Chicago, IL, USA). The significance of each group was verified with the analysis of one-way ANOVA followed by $t$-test. A $p$-value of less than 0.05 was considered significant.

\section{Results and Discussion}

Effect of UV irradiation on the absorption spectrum of quercetin The absorption spectra of quercetins before and after UV irradiation are shown in Fig. 1. Intact quercetin in methanol has a unique absorbance spectrum between 200 and $500 \mathrm{~nm}$ with two major peaks. These are seen as band I, which corresponds to the B-ring (cinnamoyl) structure, and band II, which is a result of the A-C benzoyl structure $(6,9)$. UV irradiation significantly affected the absorption spectrum of quercetin. The absorbance of UVirradiated quercetin decreased by more than $50 \%$ at both band I and band II. However, at the $300 \mathrm{~nm}$ region, the absorbance spectrum increased to an optical density of approximately 1.5 . These results indicate that the UV irradiation greatly changed the structure of quercetin. The decrease seen in the band I and band II regions might indicate changes in the B-ring and A-C benzoyl structures, respectively (6). Furthermore, the increase in optical density at the $300 \mathrm{~nm}$ region suggests the production of quercetin peroxides. The trend found in our results was similar to that reported by Zvezdanović et al. (10), where the absorption spectra of quercetin and rutin also decreased at band I and band II under UV irradiation. The possible products formed after UV-induced degradation of quercetin include the following: 2,4,6-trihydroxy-phenylglyxylic acid; 2-(3,4-dihydroxybenzoyl)-2,4,6-trihydroxy-3(2H)-benzofuranone; 1,3,8-trihydroxy-9aH,11H-benzofurol[3,2-b]-[1] benzopyran7,11-dione cyclic ether connecting the AC-system with the B-ring; and methyl 2-\{2-[(3,4-dihydroxybenzoyl)oxy]-4,6dihydroxyphenyl\}-2-oxoacetate (10).

Effect of UV irradiation on the DPPH RSA of quercetin The DPPH assay is generally used to estimate antioxidant activity by determining the free radical-scavenging ability of various samples (11). In the present study, the DPPH RSA of quercetin before and after UV irradiation was determined. As shown in Fig. 2, the DPPH RSA of $100 \mu \mathrm{M}$ quercetin significantly decreased from 86.2 to $29.0 \%$ after UV irradiation. This finding indicates that more than $65 \%$ of the DPPH RSA of intact quercetin was dissipated by UV irradiation. We conclude that UV irradiation significantly affects the antioxidant activity of quercetin due to the destruction of the B-ring and A-C benzoyl structure, which is suggested by our absorption spectrum results (Fig. 1).

The antioxidant activity related to the structure of polyphenolic compounds has been found to be dependent on the number of the active hydroxyl groups included, which means that the activity of the compound increases with increase in the number of active groups. The position of the active groups also plays an important role in structure-antioxidant activity relationship $(12,13)$. In the case of quercetin, the phenolic hydroxyl groups are responsible for its ability to scavenge DPPH and ABTS radicals (14). When the flavonol quercetin reacts with a free radical, it donates a proton and becomes a radical itself, but the resulting unpaired electron is delocalized by 


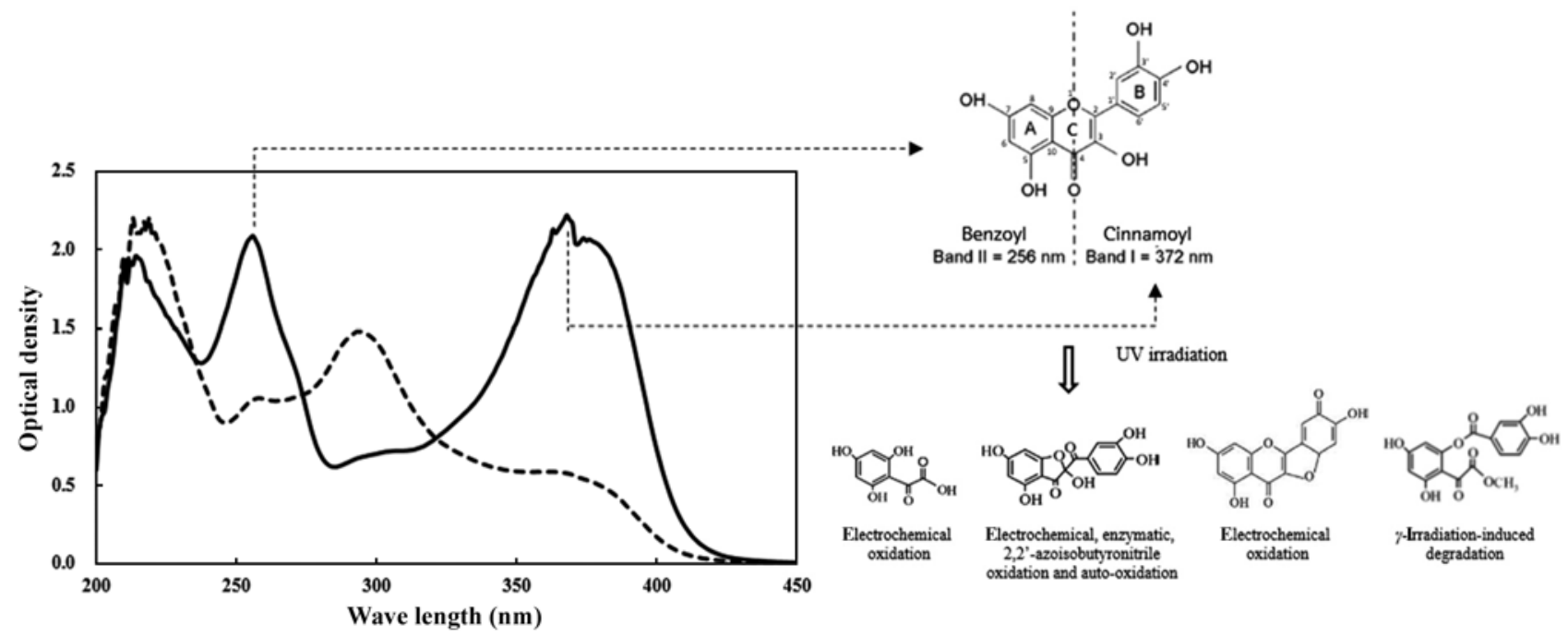

Fig. 1. Changes in the absorption spectra of intact and UV irradiated quercetins. The figure also illustrates which parts of the quercetin structure (benzoyl and cinnamoyl) contribute to spectral bands I and II (9). UV irradiation may cause the breakdown of quercetin into several different compounds (10). (-), intact quercetin; (---), UV-irradiated quercetin

resonance, thereby making the energy level of the quercetin radical too low for it to be reactive (15). Three structural groups aid in the ability of quercetin to maintain its stability and act as an antioxidant when reacting with free radicals: the B ring $o$-dihydroxyl groups, the 4-oxo group in conjugation with the 2,3-alkene, and the 3- and 5hydroxyl groups (16). The functional groups can donate electrons to the rings, which increase the number of resonance forms available in addition to those created by the benzene structure (15). In the present study, the B-ring of quercetin was degraded by UV irradiation Fig. 1 (band I), which might have resulted in the significantly decreased antioxidant activity. In a previous study (17), comparison with quercetin analogs confirmed that the hydroxyl groups in positions 3,3, and 4 are involved not only in the antioxidant activity of quercetin, but also in its photolability. Therefore, the hydroxyl groups at positions 5 and 7 of the A-ring do not play a crucial role in the phototoxidative mechanism.

Effect of UV irradiation on the anti-adipogenic activity of quercetin To test whether UV irradiation could influence the anti-adipogenic activity of quercetin, 3T3-L1 cells were used. A previous study reported that quercetin treatment decreased lipid droplets in 3T3-L1 cells and enhanced the expression of adipogenesis-related factors and enzymes via regulating the levels of phosphorylation of AMPK and its substrate (5). It has also been reported that quercetin can suppress adipogenesis via the modulation of ERK and JNK in the mitogen-activated protein kinase (MAPK) pathway by down-regulating the expression of bcl-2 and caspase-3 (5). As shown in Fig. 3, treatment with $200 \mu \mathrm{M}$ of intact and UV-irradiated quercetin significantly

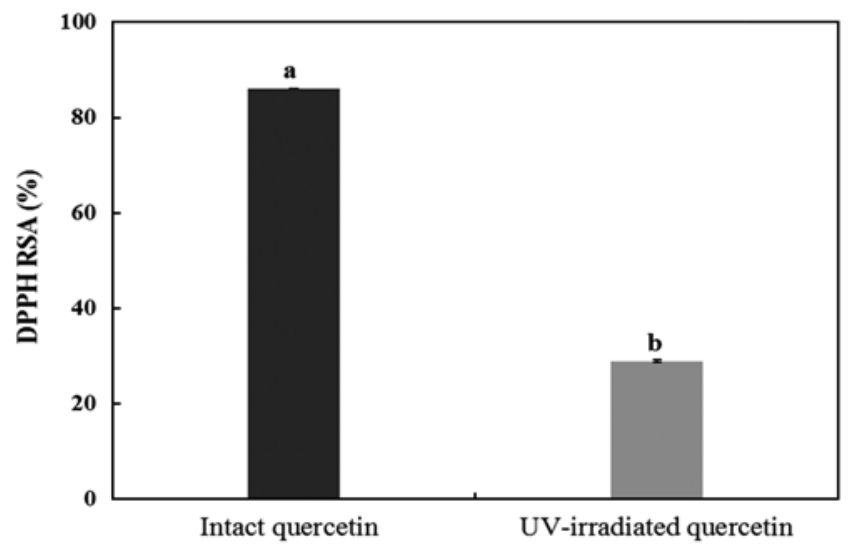

Fig. 2. Effect of UV irradiation on the scavenging activity of quercetin against DPPH radicals. Mean \pm SD values are shown. "a" and "b" are significantly different $(p<0.05)$.

suppressed the 3T3-L1 adipogenesis by 87 and $88 \%$, respectively. In addition, the size and number of lipid droplets in 3T3-L1 cell cytoplasm were decreased on treatment with both intact and UV-irradiated quercetins.

Our results show that the UV irradiation of quercetin significantly decreased its antioxidant activity. However, it exhibited little or no effect on its anti-adipogenic activity. Thus, the anti-adipogenic function (Fig. 3) was not directly correlated with the antioxidant activity of quercetin (Fig. 2). Our results indicate that quercetin efficiently suppresses 3T3-L1 adipogenesis regardless of UV irradiation. Quercetin has previously been reported to prevent intracellular lipid accumulation via hyper activation of the gene regulating lipolysis (18). Lee et al. (19) suggested that, during adipogenesis in 3T3-L1 cells, the antioxidant enzymes are positively regulated to manage oxidative stress from 

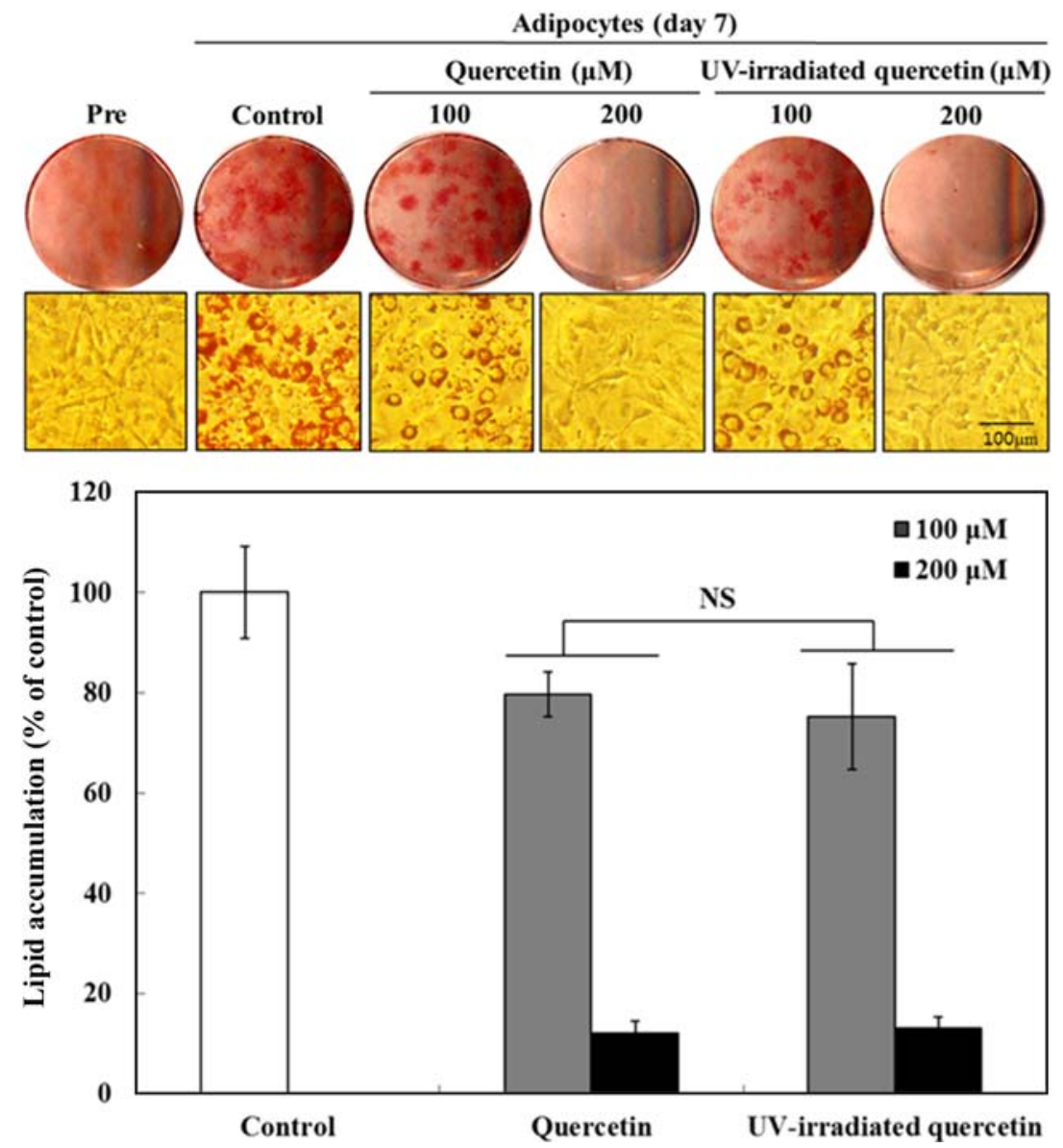

Fig. 3. Effect of UV irradiation on the anti-adipogenic activity of quercetin in 3T3-L1 cells. Oil Red O staining was performed to observe and quantify the lipid droplets after 7 days of differentiation. The bar chart represents the percentage of lipid accumulation. Pre, 3T3-L1 preadipocytes; Control, 3T3-L1 adipocytes; NS, not significant

accumulating fat, and that the anti-adipogenic action of quercetin may involve coordinated changes in the cellular redox state in adipocytes. Our findings suggested that, in the case of quercetin itself, the B-ring and A-C benzoyl structures, which are rich in hydroxyl groups, play a very important role in antioxidant activity. However, they do not seem to be involved in the anti-adipogenic activity of quercetin. Although it is still unclear how qurecetin or UVirradiated qurecetin modulated 3T3-L1 adipogenesis in molecular level, it is speculated that the quercetin could inhibit adipogenesis by regulation of early cell cycle events required for adipogenesis. In addition, quercetin-triggered AMPK activation may indirectly induce lipolytic pathways of 3T3-L1 cells. Further molecular analysis should be performed to elucidate the precise mechanism underlying the antioxidant and anti-adipogenic activities of quercetinin connection with its structure.

Acknowledgments This research was supported by Basic Science Research Program through the National Research Foundation of Korea (NRF) funded by the Ministry of
Education, Science and Technology (2012R1A1A4A01 018865).

Disclosure The authors declare no conflict of interest.

\section{References}

1. Mendoza EE, Burd R. Quercetin as a systemic chemopreventative agent: Structural and functional mechanisms. Mini Rev. Med. Chem. 11: 1216-1221 (2011)

2. Bischoff SC. Quercetin: Potentials in the prevention and therapy of disease. Curr. Opin. Clin. Nutr. 11: 733-740 (2008)

3. Hertog MG, Hollman PC. Potential health effects of the dietary flavonol quercetin. Eur. J. Clin. Nutr. 50: 63-71 (1996)

4. Wu LL, Yang XB, Huang ZM, Liu HZ, Wu GX. In vivo and in vitro antiviral activity of hyperoside extracted from Abelmoschus manihot (L) medik. Acta Pharmacol. Sin. 28: 404-409 (2007)

5. Ahn JY, Lee HJ, Kim SN, Park JH, Ha TY. The anti-obesity effect of quercetin is mediated by the AMPK and MAPK signaling pathways. Biochem. Bioph. Res. Co. 373: 545-549 (2008)

6. Cvetković D, Marković D, Cvetković D, Radovanović B. Effects of continuous UV-irradiation on the antioxidant activities of quercetin and rutin in solution in the presence of lecithin as the protective target. J. Serb. Chem. Soc. 76: 973-985 (2011) 
7. Jeong SM, Kim SY, Park HR, Lee SC. Effect of far-infrared radiation on the activity of extracts from Citrus unshiu peels. J. Korean Soc. Food Sci. Nutr. 33: 1580-1583 (2004)

8. Kim CY, Le TT, Chen C, Cheng JX, Kim KH. Curcumin inhibits adipocyte differentiation through modulation of mitotic clonal expansion. J. Nutr. Biochem. 22: 910-920 (2011)

9. Bukhari SB, Memon S, Mahroof-Tahir M, Bhanger MI. Synthesis, characterization, and antioxidant activity copper-quercetin complex. Spectrochim. Acta A 71: 1901-1906 (2009)

10. Zvezdanović JB, Stanojević JS, Marković DZ, Cvetković DJ. Irreversible UV-induced queretin and rutin degradation in solution studied by UV spectrophotometry and HPLC chromatography. J. Serb. Chem. Soc. 77: 297-312 (2012)

11. Amarowicz R, Pegg RB, Rahim-Moghaddam P, Barl B, Weil JA. Free radical-scavenging capacity and antioxidant activity of selected plant species from the Canadian praires. Food Chem. 84: 551-562 (2004)

12. Bendary E, Francis RR, Ali HMG, Sarwat MI, El Hady S. Antioxidant and structure-activity relationships (SARs) of some phenolic and anilines compounds. Ann. Agr. Sci. 58: 173-181 (2013)

13. Cai YZ, Sun M, Xing J, Luo Q, Corke H. Structure-radical scavenging activity relationships of phenolic compounds from traditional Chinese medicinal plants. Life Sci. 78: 2872-2888 (2006)

14. Zuo A, Yanying Y, Li J, Binbin X, Xiongying Y, Yan Q, Shuwen C. Study on the relation of structure and antioxidant activity of isorhamnetin, quercetin, phloretin, silybin, and phloretin isonicotinyl hydrazine. Free Radic. Antioxid. 1: 39-47 (2011)

15. Mariani C, Braca A, Vitalini S, De Tommasi N, Visioli F, Fico G. Flavonoid characterization and in vitro antioxidant activity of Aconitum anthora L. (Ranunculaceae). Phytochemistry 69: 12201226 (2008)

16. Hollman PCH, Katan MB. Absorption, metabolism, and health effects of dietary flavonoids in man. Biomed. Pharmacother. 51: 305-310 (1997)

17. Dall'Acqua S, Miolo G, Innocenti G, Caffieri S. The photodegradation of quercetin: Relation to oxidation. Molecules 17: 8898-8907 (2012)

18. Bae CR, Park YK, Cha YS. Quercetin-rich onion peel extract suppress adipogenesis by down-regulating adipogenic transcription factors and gene expression in 3T3-L1 adipocytes. J. Sci. Food Agr. 94: 2655-2660 (2014)

19. Lee OH, Swick J, O'Fallon K, Clarkson PM, Kim YC. The antiadipogenic effect of quercetin is associated with coordinated changes of antioxidant responses during adipogenic differentiation of 3T3-L1 cells. FASEB J. 23(Suppl.): 716.14 (2009) 